\title{
Testing spatial noncommutativity via the Aharonov-Bohm effect
}

\author{
H. Falomir, ${ }^{1}$ J. Gamboa, ${ }^{2}$ M. Loewe, ${ }^{3}$ F. Méndez, ${ }^{2}$ and J. C. Rojas ${ }^{2}$ \\ ${ }^{1}$ IFLP-Departamento de Física, Facultad de Ciencias Exactas, Universidad Nacional de la Plata, La Plata, Argentina \\ ${ }^{2}$ Departamento de Física, Universidad de Santiago de Chile, Casilla 307, Santiago 2, Chile \\ ${ }^{3}$ Facultad de Física, Pontificia Universidad Católica de Chile, Casilla 306, Santiago 22, Chile
}

(Received 1 April 2002; published 20 August 2002)

\begin{abstract}
The possibility of detecting noncommutative space relics is analyzed using the Aharonov-Bohm effect. We show that, if space is noncommutative, the holonomy receives nontrivial kinematical corrections that will produce a diffraction pattern even when the magnetic flux is quantized. The scattering problem is also formulated, and the differential cross section is calculated. Our results can be extrapolated to high energy physics and the bound $\theta \sim[10 \mathrm{TeV}]^{-2}$ is found. If this bound holds, then noncommutative effects could be explored in scattering experiments measuring differential cross sections for small angles. The bound state Aharonov-Bohm effect is also discussed.
\end{abstract}

DOI: 10.1103/PhysRevD.66.045018

\section{INTRODUCTION}

There are arguments in string theory suggesting that spacetime could be noncommutative [1]. Although this property might be an argument in favor of new renormalizable effective field theories [2], it represents also trouble because we need to explain the transition between the commutative and noncommutative regimes.

If the noncommutative effects are important at very high energies, then one could posit a decoupling mechanism producing the standard quantum field theory as an effective field theory having no memory about noncommutative effects. However, our experience in atomic and molecular physics [3] strongly suggests that the decoupling is never complete, and the high energy effects appear in the effective action as topological remnants [4].

Following this idea we would like to consider an example, related to topological aspects, where the appearance of noncommutative effects could be relevant. A natural candidate is the Aharonov-Bohm effect [5] where, as we know, the relativistic corrections do not change the qualitative behavior of the fringe pattern [6].

As we will see, if the space is noncommutative the total holonomy contains - as we will show below-a term dependent on the velocity of the electrons, which tends to shift the line spectrum. Moreover, a new effect is produced by noncommutativity: Particles are scattered even when the magnetic flux is quantized.

Our conclusions are reinforced by studying the bound state Aharonov-Bohm effect [7]. In this case, although the Schrödinger equation cannot be exactly solved, one can extract information through perturbation theory since $\theta<<1$.

As a bonus of the previous results, one finds - using perturbation theory - an explicit expression for the scattering amplitude and a formula for the differential cross section of the noncommutative Aharonov-Bohm effect.

There is, however, another interesting conclusion that can be extracted from our research. The quantum mechanical Aharonov-Bohm effect is also a relevant mechanism to explain other high energy phenomena. In this sense, our calculations allow us to extract conclusions for other high energy processes, e.g., cosmic strings and grand unfied theories
PACS number(s): 03.65.- $-\mathrm{w}$

(GUTs) [8]. More precisely, using our noncommutative differential cross section, we are able to find a bound for the theta parameter which is in full agreement with other estimations [9].

The paper is organized as follows: in Sec. II, the noncommutative Aharonov-Bohm effect is discussed and a formula for the holonomy is derived; in Sec. III we explain the noncommutative corrections to the bound state Aharonov-Bohm effect. The general Schrödinger equation and the scattering problem in a noncommutative space are considered in Sec. $\mathrm{IV}$; in Sec. V, we study the first order noncommutative corrections to the scattering amplitude; in Sec. VI, we estimate a bound for the noncommutative parameter and we analyze the experimental possibilities for detecting noncommutative relics and, finally, Sec. VII contains the conclusions. Two Appendixes containing a discussion on the commutative Aharonov-Bohm effect at high energy and some technical details are included.

\section{THE NONCOMMUTATIVE AHARONOV-BOHM EFFECT}

\section{A. The Schrödinger equation}

In the commutative case, the Schrödinger equation with an external gauge potential is solved by

$$
\psi=e^{i \int_{C} d x^{j} A_{j}} \varphi
$$

where $\varphi$ is the solution of the free Schrödinger equation, and the $U(1)$ holonomy, $e^{i \int_{C} d x^{j} A_{j}}$, is in general a non-integrable factor, i.e., it depends on the integration path $C$.

Although Eq. (1) solves formally the Schrödinger equation, the holonomy involves in a nontrivial way the dynamics of the gauge potential, hiding all the complications related to A. Our goal below will be to find an approximate expression for the holonomy, valid for small values of the fundamental noncommutative parameter $\theta$.

In the following we assume that wave functions in the plane belong to a noncommutative algebra characterized by the Moyal product, defined as

$$
(\mathbf{A} \star \mathbf{B})(\mathbf{x})=\left.e^{i / 2 \theta \epsilon^{i j} \partial_{i}^{(1)} \partial_{j}^{(2)}} \mathbf{A}\left(\mathbf{x}_{1}\right) \mathbf{B}\left(\mathbf{x}_{2}\right)\right|_{\mathbf{x}_{1}=\mathbf{x}_{2}=\mathbf{x}} .
$$



is

The Schrödinger equation in this noncommutative space

$$
\hat{H} \star \psi=\frac{1}{2 m} D_{j} \star D_{j} \star \psi=\frac{1}{2 m} k_{j} k_{j} \psi,
$$

where $k_{j}$ are the eigenvalues of the operator $D_{j}=-i \partial_{j}$ $+A_{j}$, i.e.,

$$
D_{j} \star \psi=k_{j} \psi,
$$

we are assuming, of course, that the magnetic field is zero everywhere except in the origin.

In order to solve Eq. (4) we use the ansatz

$$
\psi=e^{F}
$$

As we are assuming that noncommutative effects are small, we expand the Moyal product retaining only linear terms in $\theta$,

$$
\begin{aligned}
D_{j} \star \psi= & -i \partial_{j} e^{F}+A_{j} \star e^{F} \\
= & e^{F}\left[-i \partial_{j} F+A_{j}+\frac{i}{2} \theta \epsilon^{l m}\left(\partial_{l} A_{j}\right)\right. \\
& \left.\times\left(\partial_{m} F\right)\right] .
\end{aligned}
$$

Then Eq. (4) becomes

$$
-i \partial_{j} F+A_{j}+\frac{i}{2} \theta \epsilon^{l m}\left(\partial_{l} A_{j}\right)\left(\partial_{m} F\right)=k_{j}
$$

Now, one can solve Eq. (6) perturbatively expanding $F$ and $A_{j}$ in powers of $\theta$, i.e.,

$$
\begin{gathered}
F=F^{(0)}+\theta F^{(1)}+\cdots \\
A_{j}=A_{j}^{(0)}+\theta A_{j}^{(1)}+\cdots .
\end{gathered}
$$

At zero order in $\theta$, Eq. (6) gives

$$
-i \partial_{j} F^{(0)}+A_{j}^{(0)}=k_{j},
$$

from which the following expression for $F^{(0)}$ is obtained:

$$
F^{(0)}=i k_{j}\left(x-x_{0}\right)_{j}-i \int_{x_{0}}^{x} d x_{j} A_{j}^{(0)} .
$$

The first term in the right-hand side (RHS) is just the free particle solution if we interpret $k_{j}$ as the wave number, and the second term is the $U(1)$ holonomy for the commutative case. Thus, at zero order we reproduce the solution of the commutative case Schrödinger equation.

If we retain first order terms in $\theta$, the following differential equation is obtained:

$$
-i \partial_{j} F^{(1)}+A_{j}^{(1)}+\frac{i}{2} \epsilon^{l m}\left(\partial_{l} A_{j}^{(0)}\right)\left(\partial_{m} F^{(0)}\right)=0,
$$

which by integration gives

$$
\begin{aligned}
F^{(1)}= & -i \int_{x_{0}}^{x} d x_{j} A_{j}^{(1)}-\frac{i}{2} \int_{x_{0}}^{x} d x_{j} \epsilon^{m l} k_{m} \partial_{l} A_{j}^{(0)} \\
& +\frac{i}{2} \int_{x_{0}}^{x} d x_{j} \epsilon^{m l} A_{m}^{(0)} \partial_{l} A_{j}^{(0)} .
\end{aligned}
$$

The first term in the RHS of Eq. (12) is an additive correction to the commutative holonomy which, together with the second term in the RHS of Eq. (10) gives

$$
-i \int_{x_{0}}^{x} d x_{j}\left(A_{j}^{(0)}+\theta A_{j}^{(1)}\right) .
$$

The second in the RHS of Eq. (12) is a velocity dependent term, which can be written as [10-12]

$$
-\frac{i}{2} \int_{x_{0}}^{x} d x_{j} \epsilon^{m l} k_{m} \partial_{l} A_{j}^{(0)}=-\frac{i}{2} m \int_{x_{0}}^{x} d x_{j}\left(\mathbf{v} \times \nabla A_{j}^{(0)}\right)_{3} .
$$

For the last term our calculation yields

$$
\frac{i}{2} \int_{x_{0}}^{x} d x_{j} \epsilon^{m l} A_{m}^{(0)} \partial_{l} A_{j}^{(0)}=\frac{i}{2} \int_{x_{0}}^{x} d x_{j}\left(\mathbf{A}^{(0)} \times \nabla A_{j}^{(0)}\right)_{3} .
$$

Thus, at this order in $\theta$, the nonconmutative holonomy is given by

$$
\begin{aligned}
\mathcal{W}\left(x, x_{0}\right)= & \exp \left\{-i\left[\int_{x_{0}}^{x} d x_{j} A_{j}+\frac{\theta}{2} \int_{x_{0}}^{x} d x_{j}\left[m\left(\mathbf{v} \times \nabla A_{j}^{(0)}\right)_{3}\right.\right.\right. \\
& \left.\left.\left.-\left(\mathbf{A}^{(\mathbf{0})} \times \boldsymbol{\nabla} A_{j}^{(0)}\right)_{3}\right]\right]\right\}
\end{aligned}
$$

Now, we analyze the different terms in Eq. (16). The first one in the exponential is the usual holonomy, corrected to order $\theta$, which classifies the different homotopy classes. The term in Eq. (15) is a noncommutative correction to the vortex decaying as $1 / r^{3}$, which does not contribute to the line spectrum. Finally, the term in Eq. (14) is a velocity dependent correction insensitive to the topology of the manifold.

In the commutative Aharonov-Bohm effect, the presence of the flux produces a shift in the interference pattern, which is maximum for $\Phi=(2 n+1) \pi(\hbar c / e)$, with $n \in Z$. In such a case, for a given value of $n$, the position of maxima and minima are interchanged due to a change of $\pi$ in the phase. However, in the noncommutative case, this change of positions of maxima and minima might not occur. Indeed, the velocity dependent correction modifies the phase shift which, for suitable values of velocity, could even become $2 \pi$ for a given $n$.

We finalize this section emphasizing two importants aspects of our results:

The above results are a general property of the noncommutative Aharonov-Bohm effect, depending only on the total flux $\Phi$ (if the electrons cannot penetrate into the solenoid).

If the magnetic flux $e \Phi / h c$ is an integer there is no Aharonov-Bohm effect for the commutative case, as is well known $[5,13]$. However, in the noncommutative case the 
term (14) is different from zero even in the case where $e \Phi / h c$ is an integer. This is a quite nontrivial characteristic of the noncommutative Aharonov-Bohm effect that could be experimentally measured.

\section{B. The gauge potentials}

In this section we will evaluate the gauge potential for a finite radius solenoid orthogonal to a noncommutative plane.

The field tensor in the noncommutative plane is

$$
\hat{F}_{\mu \nu}=\partial_{\mu} A_{\nu}-\partial_{\nu} A_{\mu}+i A_{\mu} \star A_{\nu}-i A_{\nu} \star A_{\mu} .
$$

Expanding the Moyal product and retaining only the linear term in $\theta$, we have

$$
\hat{F}_{\mu \nu}=\partial_{\mu} A_{\nu}-\partial_{\nu} A_{\mu}+\theta \epsilon^{\alpha \beta} \partial_{\alpha} A_{\mu} \partial_{\beta} A_{\nu} .
$$

We must construct a gauge potential such that the magnetic field $B_{3}=\hat{F}_{12}$ vanishes everywhere, except inside the solenoid. We proceed as in the commutative case, starting with the ansatz (where the ordinary product is understood)

$$
\begin{aligned}
& A_{1}=-x_{2} f\left(r^{2}\right), \\
& A_{2}=x_{1} f\left(r^{2}\right),
\end{aligned}
$$

for $r>a$, the radius of the solenoid.

We impose the condition $B_{3}=\hat{F}_{12}=0$ outside the solenoid, implying that

$$
2 f+2 r^{2} f^{\prime}+\theta\left(f^{2}+2 r^{2} f f^{\prime}\right)=0,
$$

where $f^{\prime}=d f / d r^{2}$.

This differential equation can be easily integrated, yielding the following solutions:

$$
\begin{aligned}
f & =-\frac{1}{\theta} \pm \frac{1}{\theta} \sqrt{1+\frac{c_{1} \theta}{r^{2}}} \\
& =-\frac{1}{\theta} \pm \frac{1}{\theta}\left[1+\frac{c_{1} \theta}{2 r^{2}}-\frac{c_{1}^{2} \theta^{2}}{8 r^{4}}+\cdots\right],
\end{aligned}
$$

where $c_{1}$ is an integration constant.

From Eq. (21) we see that the commutative limit is smooth for the plus sign in the above equation, then we adopt

$$
f=\frac{c_{1}}{2 r^{2}}-\frac{c_{1}^{2} \theta}{8 r^{4}}+\cdots
$$

We determine the integration constant by imposing the Stokes theorem at zero order in $\theta$,

$$
\int \vec{B} \cdot d \vec{S}=\Phi=\oint \vec{A}^{(0)} \cdot d \vec{l}
$$

getting

$$
c_{1}=\frac{\Phi}{\pi}=B a^{2}
$$

Notice that Eq. (21) requires $B \theta \ll 1$.

The final expression for the gauge potential becomes

$$
\begin{aligned}
& A_{1}=x_{2}\left(\frac{\Phi}{2 \pi\left(x_{1}^{2}+x_{2}^{2}\right)}-\theta \frac{\Phi^{2}}{8 \pi^{2}\left(x_{1}^{2}+x_{2}^{2}\right)^{2}}+\cdots\right), \\
& A_{2}=-x_{1}\left(\frac{\Phi}{2 \pi\left(x_{1}^{2}+x_{2}^{2}\right)}-\theta \frac{\Phi^{2}}{8 \pi^{2}\left(x_{1}^{2}+x_{2}^{2}\right)^{2}}+\cdots\right),
\end{aligned}
$$

where $\Phi=B \pi a^{2}$ is the magnetic flux enclosed into the solenoid.

We finally give the gauge potential expressed in terms of polar coordinates,

$$
\begin{aligned}
& A_{r}=0, \\
& A_{\varphi}=\frac{\Phi}{2 \pi r}-\theta \frac{\Phi^{2}}{8 \pi^{2} r^{3}}+\mathcal{O}\left(\theta^{2}\right),
\end{aligned}
$$

which will be useful in solving the Schrödinger equation in the next sections.

\section{BOUND STATES FOR THE NONCOMMUTATIVE AHARONOV-BOHM EFFECT}

In this section we will solve the noncommutative Schrodinger equation (3) for an electron moving in a twodimensional manifold parametrized by polar coordinates $(r, \varphi)$, with $r>a$ and $0<\varphi<2 \pi$.

Before doing this, it is necessary to explain an important technical point: The Moyal product (2) is implicitly written in Cartesian coordinates. Therefore, in order to solve Eq. (3) in polar coordinates, one must express the $\star$ product in the general case.

We find the following expression for the Moyal product up to first order in $\theta$ :

$$
f(x) \star p(x)=f(x) p(x)+\frac{i \theta}{2 \sqrt{g}} \epsilon^{\mu \nu} \partial_{\mu} f \partial_{\nu} p+\mathcal{O}\left(\theta^{2}\right),
$$

where $g$ is the determinant of the metric.

At this order in $\theta$, the Schrodinger equation becomes

$$
\left(\hat{H}_{0}+\theta \hat{H}_{1}\right) \Psi=k^{2} \Psi
$$

where $H_{0}$ and $H_{1}$ can be identified by replacing Eq. (25) in Eq. (3), and taking into account that the covariant derivative becomes

$$
\vec{D}=-i\left[\hat{r} \partial_{r}+\hat{\varphi}\left(\frac{\partial_{\varphi}}{r}-i A_{\varphi}\right)\right]
$$

We get 


$$
\begin{aligned}
\hat{H}_{0}= & \partial_{r}^{2}+\frac{1}{r} \partial_{r}+\frac{1}{r^{2}}\left(\partial_{\varphi}^{2}-i \frac{\Phi}{\pi} \partial_{\varphi}-\frac{\Phi^{2}}{4 \pi^{2}}\right) \\
\hat{H}_{1}= & \frac{1}{r^{3}}\left(-i \partial_{\varphi}-\frac{\Phi}{4 \pi}\right) \partial_{r}+\frac{1}{r^{4}}\left(-i \partial_{\varphi}^{3}-\frac{\Phi}{\pi} \partial_{\varphi}^{2}\right. \\
& \left.+i \frac{\Phi^{2}}{2 \pi^{2}} \partial_{\varphi}+\frac{\Phi^{3}}{8 \pi^{3}}\right) .
\end{aligned}
$$

As $\theta$ is very small, one can use perturbation theory for computing the eigenvalues and eigenfunctions of the Hamiltonian $\hat{H}=\hat{H}_{0}+\theta \hat{H}_{1}$.

In the following subsections, we find explicitly the energy spectrum for the bound state Aharonov-Bohm effect.

\section{A. The noncommutative bound state Aharonov-Bohm effect}

The bound state Aharonov-Bohm effect is a result due to Peshkin et al. [7], which establishes the flux and angular momentum dependence of the energy spectrum, a measurable quantity in principle. In this effect one considers an electron constrained to move between two impenetrable concentric cylinders with outer and inner radius $b$ and $a$, respectively, and in the presence of a magnetic flux $\Phi$ contained inside the inner one.

In the noncommutative space, the Schrödinger radial equation at first order in $\theta$ is given by

$$
\begin{aligned}
& -\chi_{\ell}^{\prime \prime}(r)-\left(\frac{1}{r}+\frac{\theta\left(\ell-\frac{\Phi}{4 \pi}\right)}{r^{3}}\right) \chi_{\ell}^{\prime}(r)+\left(\frac{\left(\ell-\frac{\Phi}{2 \pi}\right)^{2}}{r^{2}}\right. \\
& \left.+\frac{\theta\left(\ell^{3}-\frac{\ell^{2} \Phi}{\pi}+\frac{\ell \Phi^{2}}{2 \pi^{2}}-\frac{\Phi^{3}}{8 \pi^{3}}\right)}{r^{4}}\right) \chi_{\ell}(r) \\
& =\left(\hat{h}_{\ell, 0}+\theta \hat{h}_{l, 1}\right) \chi_{\ell}(r)=k^{2} \chi_{\ell}(r),
\end{aligned}
$$

where we have called

$$
\begin{aligned}
\hat{h}_{\ell, 0}= & -\partial_{r}^{2}-\frac{1}{r} \partial_{r}+\frac{1}{r^{2}}\left(\ell-\frac{\Phi}{2 \pi}\right)^{2}, \\
\hat{h}_{\ell, 1}= & -\frac{1}{r^{3}}\left(\ell-\frac{\Phi}{4 \pi}\right) \partial_{r}+\frac{1}{r^{4}}\left(\ell^{3}-\frac{\ell^{2} \Phi}{\pi}\right. \\
& \left.+\frac{\ell \Phi^{2}}{2 \pi^{2}}-\frac{\Phi^{3}}{8 \pi^{3}}\right),
\end{aligned}
$$

and the following ansatz has been used for the wave function:

$$
\psi(r, \varphi)=\sum_{\ell \in Z} e^{i \ell \varphi} \chi_{\ell}(r)
$$

Although this equation cannot be solved exactly, one can use perturbation theory in the small parameter $\theta$. Since noncommutative effects are important only at small distances $\sim \sqrt{\theta}$, one would expect some relevant consequences in the high energy region, $k \sim 1 / \sqrt{\theta}$.

Equation (31) contains the commutative Aharonov-Bohm effect as a particular case, for $\theta=0$. The 0 th order solution can be written as

$$
\chi_{\ell}(r)=A_{\ell} J_{\nu}(k r)+B_{\ell} Y_{\nu}(k r),
$$

with $\nu=|\ell-\Phi / 2 \pi|$. The constants $A_{\ell}, B_{\ell}$ and the Hamiltonian eigenvalues $E_{\ell, 0}=k^{2}$ can be obtained-as usual-by imposing the boundary conditions on $\chi_{\ell}(r)$,

$$
\chi_{\ell}(a)=0=\chi_{\ell}(b),
$$

together with the normalization condition for the eigenfunction,

$$
\int_{a}^{b} \chi_{\ell}(r)^{2} r d r=1
$$

Notice that the eigenvalues depend on the angular momentum $\ell$ only through $\nu$. Therefore, degeneracy will occur if $\left|\ell_{1}-\Phi / 2 \pi\right|=\left|\ell_{2}-\Phi / 2 \pi\right|$, which is possible only if $\Phi / \pi$ is an integer. For simplicity, to be able to apply perturbation theory in its simplest form, in this section we will avoid these particular values of the flux.

Taking into account Eq. (36), the mean value of $\hat{h}_{\ell, 1}$ can be straightforwardly cast in the form

$$
E_{\ell, 1}=\int_{a}^{b} \chi_{\ell}(r) \hat{h}_{\ell, 1} \chi_{\ell}(r) r d r=P(\ell, \Phi)\left\langle r^{-4}\right\rangle_{\ell},
$$

where $P(\ell, \Phi)$ is a cubic polynomial,

$$
P(\ell, \Phi)=\left[\ell^{3}-\frac{\ell^{2} \Phi}{\pi}+\ell\left(\frac{\Phi^{2}}{2 \pi^{2}}-1\right)+\frac{2 \pi^{2} \Phi-\Phi^{3}}{8 \pi^{3}}\right],
$$

and

$$
\left\langle r^{-4}\right\rangle_{\ell}=\int_{a}^{b} \frac{\chi_{\ell}^{2}(r)}{r^{4}} r d r
$$

is a function of $\nu, a, b$ and $k$ only.

Since noncommutative effects are expected to occur at high energies $(k a \gg 1)$, it is enough to use in Eq. (35) the first terms in the asymptotic expansions of Bessel functions for large arguments. We will retain just the first two terms in these expansions, i.e.,

$$
\begin{aligned}
& J_{\nu}(z) \rightarrow \sqrt{\frac{2}{z \pi}}\left[\cos \left(z-\frac{\pi \nu}{2}-\frac{\pi}{4}\right)-\frac{4 \nu^{2}-1}{8 z}\right. \\
& \left.\times \sin \left(z-\frac{\pi \nu}{2}-\frac{\pi}{4}\right)\right], \\
& Y_{\nu}(z) \rightarrow-\sqrt{\frac{2}{z \pi}}\left[\sin \left(z-\frac{\pi \nu}{2}-\frac{\pi}{4}\right)+\frac{4 \nu^{2}-1}{8 z}\right. \\
& \left.\times \cos \left(z-\frac{\pi \nu}{2}-\frac{\pi}{4}\right)\right] \text {. }
\end{aligned}
$$

Using Eq. (41) in Eqs. (37) and (40), we get 


$$
\begin{aligned}
\left\langle r^{-4}\right\rangle_{\ell}= & \frac{128 a^{3} k^{5}}{D(\nu, a, b, k)} \times\left\{-\frac{1}{768 a^{6} b^{3} k^{4}}\left(512 a^{5} k^{2}+8 a^{3}\left(1-4 \nu^{2}\right)^{2}+128 a^{4} b^{3} k^{4}\left(4 \nu^{2}-9\right)+b^{3}\left(1-4 \nu^{2}\right)^{2}\left(7+4 \nu^{2}\right)\right.\right. \\
& \left.+\left(2 a^{2} b^{3} k^{2}\right)\left(116 \nu^{2}+48 \nu^{4}-64 \nu^{6}-31\right)\right)-\frac{\cos [2(a-b) k]}{768 a^{3} b^{4} k^{4}}\left(b\left(2 b^{2} k^{2}-1\right)\left(1-4 \nu^{2}\right)^{2}-64 a^{2} b k^{2}\left(4 \nu^{2}-9\right)\right. \\
& \left.\times\left(2 b^{2} k^{2}-1\right)+8 a\left(4 \nu^{2}-1\right)\left[3-12 \nu^{2}+2 b^{2} k^{2}\left(4 \nu^{2}-9\right)\right]\right)+\frac{\sin [2 k(a-b)]}{1536 a^{3} b^{4} k^{5}}\left(( 1 - 4 \nu ^ { 2 } ) ^ { 2 } \left[3-12 \nu^{2}+2 b^{2} k^{2}\right.\right. \\
& \left.\left.\times\left(4 \nu^{2}-9\right)\right]-64 a^{2} k^{2}\left[3-12 \nu^{2}+2 b^{2} k^{2}\left(4 \nu^{2}-9\right)\right]-32 a b k^{2}\left(-1+2 b^{2} k^{2}\right)\left(9-40 \nu^{2}+16 \nu^{4}\right)\right) \\
& +\operatorname{Ci}(2 a k)\left(\cos (2 k a)\left[\frac{16 \nu^{4}-40 \nu^{2}+9}{12 a^{2}}\right]+\sin (2 k a)\left[\frac{\left(4 \nu^{2}-9\right)\left[64 a^{2} k^{2}-\left(1-4 \nu^{2}\right)^{2}\right]}{192 a^{3} k}\right]\right)+\operatorname{Ci}(2 b k)(\sin (2 k a) \\
& \left.\times\left[\frac{\left(4 \nu^{2}-9\right)\left[-64 a^{2} k^{2}+\left(1-4 \nu^{2}\right)^{2}\right]}{192 a^{3} k}\right]-\cos (2 k a)\left[\frac{16 \nu^{4}-40 \nu^{2}+9}{12 a^{2}}\right]\right)+\operatorname{Si}(2 a k)\left(\sin (2 k a) \frac{16 \nu^{4}-40 \nu^{2}+9}{12 a^{2}}\right. \\
& \left.+\cos (2 k a) \frac{\left(4 \nu^{2}-9\right)\left[-64 a^{2} k^{2}+\left(1-4 \nu^{2}\right)^{2}\right]}{192 a^{3} k}\right)+\operatorname{Si}(2 b k)\left(\cos (2 k a) \frac{\left(4 \nu^{2}-9\right)\left[64 a^{2} k^{2}-\left(1-4 \nu^{2}\right)^{2}\right]}{192 a^{3} k}\right. \\
& \left.\left.-\sin (2 k a) \frac{16 \nu^{4}-40 \nu^{2}+9}{12 a^{2}}\right)\right\},
\end{aligned}
$$

where

$$
\begin{aligned}
D(\nu, a, b, k)= & 8 k\left(-4 a\left(-1+4 \nu^{2}\right)-\frac{(a-b)\left[64 a^{2} k^{2}+\left(1-4 \nu^{2}\right)^{2}\right]}{2}+4 a\left(-1+4 \nu^{2}\right) \cos [2(a-b) k]\right. \\
& \left.+\frac{\left[64 a^{2} k^{2}-\left(1-4 \nu^{2}\right)^{2}\right] \sin [2(a-b) k]}{4 k}\right)+\left(4 \nu^{2}-1\right)\left[\operatorname { C i } ( 2 a k ) \left(16 a k\left(-1+4 \nu^{2}\right) \cos (2 a k)+\left[64 a^{2} k^{2}-(1\right.\right.\right. \\
& \left.\left.\left.-4 \nu^{2}\right)^{2}\right] \sin (2 a k)\right)+\operatorname{Ci}(2 b k)\left(-16 a k\left(4 \nu^{2}-1\right) \cos (2 a k)+\left[-64 a^{2} k^{2}+\left(1-4 \nu^{2}\right)^{2}\right] \sin (2 a k)\right)-[\operatorname{Si}(2 a k) \\
& \left.-\operatorname{Si}(2 b k)]\left[\left(64 a^{2} k^{2}-\left(1-4 \nu^{2}\right)^{2}\right) \cos (2 a k)+16 a k\left(1-4 \nu^{2}\right) \sin (2 a k)\right]\right]
\end{aligned}
$$

and

$$
\begin{aligned}
& \operatorname{Ci}(\mathrm{z})=-\int_{z}^{\infty} \frac{\cos (t)}{t} d t, \\
& \operatorname{Si}(\mathrm{z})=\int_{0}^{z} \frac{\sin (t)}{t} d t .
\end{aligned}
$$

Despite this aspect, $\left\langle r^{-4}\right\rangle$ is a slowly varying function of $\nu$, as can be seen in Fig. 1. Moreover, for a given $\nu,\left\langle r^{-4}\right\rangle$ rapidly approaches a constant value when $k$ grows up, as shown in Fig. 2.

Consequently, it is the coefficient of $\left\langle r^{-4}\right\rangle$ in Eq. (38), the cubic polynomial $P(\ell, \Phi)$, which governs the shift produced on the eigenvalues. Notice that, for given flux $\Phi$ and angular momentum $\ell$, the successive (large) eigenvalues of the radial equation (31) are all shifted by the same constant. In particular, for large $|\ell|$, this constant does not change sign.
Therefore, even though the 0th order spectrum depends only on $\nu=|\ell-\Phi / 2 \pi|$, the first order $\left(\theta^{1}\right)$ correction depends separately on the flux $\Phi$ and the angular momentum $\ell$, introducing a shift in the eigenvalues sensitive to the sign of $\ell$.

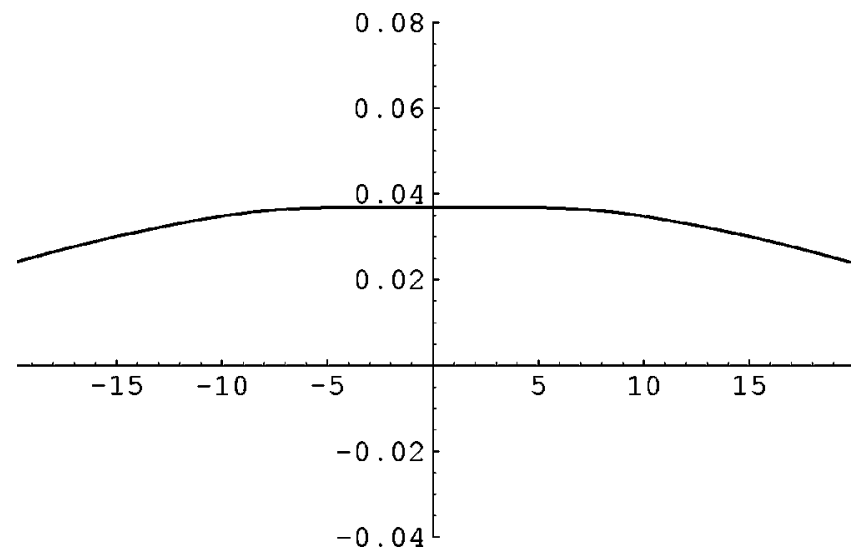

FIG. $1 .\left\langle r^{-4}\right\rangle_{\ell}$ as a function of $\nu$, for $b / a=10$ and $k a=40$. 


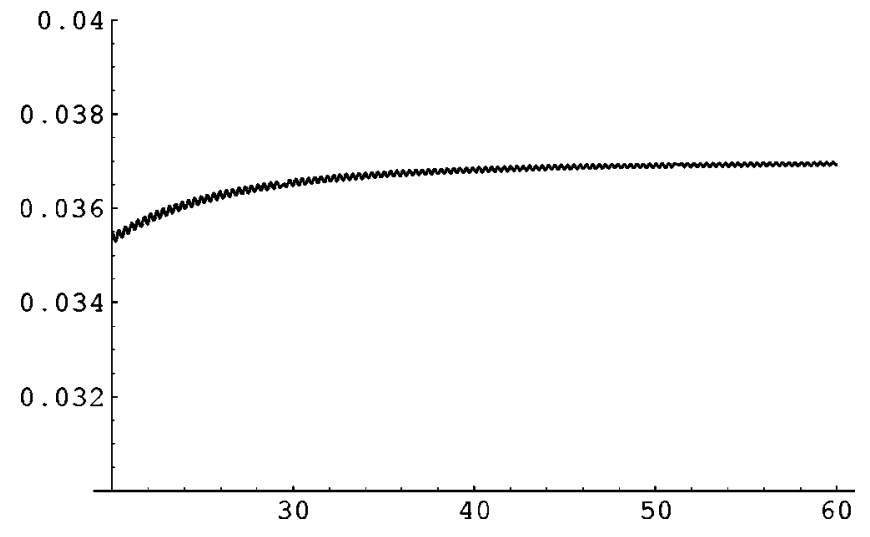
$-\sqrt{\pi}$

FIG. 2. $\left\langle r^{-4}\right\rangle_{\ell}$ as a function of $k a$, for $b / a=10$ and $\nu=7$

Finally, we would like to add some comments related to the relativistic case. Although in this paper we solve the Schrödinger equation, our conclusions are valid in the relativistic case too, indeed, as the Aharonov-Bohm interaction is static, the Schrödinger and Klein-Gordon equations are related by $E_{S c h} \rightarrow E_{K G}^{2}-m^{2}$. However, a delicate point is the following: as we are thinking in electrons, one should use the Dirac equation instead of the Schrodinger one. In such a case there is a critical subspace which admits nontrivial selfadjoint extensions [14-16].

In our case the boundary conditions ensure that the eigenfunctions have a finite limit for $r \rightarrow 0$. This could correspond to a possible self-adjoint extension. In any case, for first order corrections, as we have done, everything is consistent. For the perturbation (33), the problem is defined for $r \geqslant a$, which ruled out the case $r=0$. In spite of this constraint, one can consider the case $a \rightarrow 0$, but the boundary condition (36) ensures the self-adjoint properties, as, e.g., in quantum mechanics.

\section{SCATTERING STATES FOR THE NONCOMMUTATIVE AHARONOV-BOHM EFFECT}

\section{A. The perturbative solution}

In order to compute the scattering states we look for solutions of Eq. (27) in the form

$$
\Psi=\Psi_{0}+\theta \Psi_{1}+\cdots
$$

implying that

$$
\begin{gathered}
\hat{H}_{0} \Psi_{0}=k^{2} \Psi_{0}, \\
\left(\hat{H}_{0}-k^{2}\right) \Psi_{1}=-\hat{H}_{1} \Psi_{0} .
\end{gathered}
$$

Therefore, the correction to the wave function at first order in perturbation theory results in

$$
\Psi_{1}(r, \varphi)=-\left(\hat{H}_{0}-k^{2}\right)^{-1} \hat{H}_{1} \Psi_{0}
$$

where the 0th order wave function satisfies the boundary conditions

$$
\begin{gathered}
\Psi_{0}(a, \varphi)=0 \\
\Psi_{0}(r \rightarrow \infty, \varphi) \sim e^{i k r \cos (\varphi)}+f(\varphi, k) \frac{e^{i k r}}{\sqrt{r}}
\end{gathered}
$$

The first equation guarantees that the electron never reaches the region $r<a$, while the second one is the usual scattering condition.

The formal solution in Eq. (47) is given by

$$
\Psi_{1}(\mathbf{x})=-\int d \mathbf{x}^{\prime} G\left(\mathbf{x}, \mathbf{x}^{\prime}\right) \widehat{H_{1}} \Psi_{0}\left(\mathbf{x}^{\prime}\right),
$$

where $G\left(x, x^{\prime}\right)$ is the Green function of the unperturbed problem, that is

$$
\left(\hat{H}_{0}-k^{2}\right) G\left(r, \varphi ; r^{\prime}, \varphi^{\prime}\right)=\frac{1}{r} \delta\left(r-r^{\prime}\right) \delta\left(\varphi-\varphi^{\prime}\right)
$$

\section{B. The Green function}

We propose a solution for Eq. (50) of the form

$$
G\left(r, \varphi ; r^{\prime}, \varphi^{\prime}\right)=\frac{1}{2 \pi} \sum_{\ell \in Z} e^{i \ell\left(\varphi-\varphi^{\prime}\right)} g_{\ell}\left(r, r^{\prime}\right) .
$$

Replacing this in Eq. (50) and using an appropriate representation for the delta function, we obtain

$$
\left(\hat{h}_{\ell, 0}-k^{2}\right) g_{\ell}\left(r, r^{\prime}\right)=\frac{1}{r} \delta\left(r-r^{\prime}\right),
$$

where $g_{\ell}\left(r, r^{\prime}\right)$ must also satisfy the appropriate boundary conditions,

$$
g_{\ell}\left(a, r^{\prime}>a\right)=0, \quad g_{\ell}\left(r \rightarrow \infty, r^{\prime}\right) \sim \frac{e^{i k r}}{\sqrt{k r}} .
$$

For $r \neq r^{\prime}$, Eq. (52) is just the Schrödinger equation for the commutative Aharonov-Bohm effect, whose solutions are linear combinations of Bessel functions, as in Eq. (35). Let us introduce two linearly independent solutions of this homogeneous equation, satisfying the boundary condition at $r$ $=a$ and $r \rightarrow \infty$, respectively,

$$
\begin{aligned}
& \chi_{\ell}^{(a)}(r)=Y_{\nu}(k a) J_{\nu}(k r)-Y_{\nu}(k r) J_{\nu}(k a), \\
& \chi_{\ell}^{(\infty)}(r)=J_{\nu}(k r)+i Y_{\nu}(k r)=H_{\nu}^{(1)}(k r),
\end{aligned}
$$

where $H_{\nu}^{(1)}(z)$ is the Hankel functions.

The continuity of $g_{\ell}\left(r, r^{\prime}\right)$ at $r=r^{\prime}$, together with the discontinuity in its first derivative implied by the RHS of Eq. (52), lead to

$$
g_{\ell}\left(r, r^{\prime}\right)=C_{0} \begin{cases}\chi_{\ell}^{a}(r) \chi_{\ell}^{(\infty)}\left(r^{\prime}\right), & r<r^{\prime} \\ \chi_{\ell}^{a}\left(r^{\prime}\right) \chi_{\ell}^{(\infty)}(r) & r>r^{\prime}\end{cases}
$$

where the constant $C_{0}$ is given by 


$$
C_{0}=\frac{1}{r W\left[\chi_{\ell}^{(a)}(r), \chi_{\ell}^{(\infty)}(r)\right]},
$$

with $W[f, g]=f g^{\prime}-f^{\prime} g$ being the Wronskian.

\section{The free solution and the commutative case scattering theory}

The last ingredient we need for computing Eq. (49) is to express appropriately $\Psi_{0}$ satisfying the boundary conditions (48). We write

$$
\Psi_{0}(r, \varphi)=\sum_{\ell \in Z} e^{i \ell \varphi} \chi_{\ell}^{(0)}(r)
$$

with

$$
\chi_{\ell}^{(0)}(r)=\left[A_{\ell} J_{\nu}(k r)+B_{\ell} Y_{\nu}(k r)\right] .
$$

For convenience, in what follows we will develop a partial waves analysis of the scattering amplitude, as in [13]. There are other treatments of this problem in the literature (see, for example, [5,17] and [18]) leading to results differing in the forward scattering term, but having the same scattering amplitude for nonvanishing angles. This justifies our approach to the cross section for $\varphi \neq 0$.

The first condition in Eq. (48) implies that

$$
A_{l} J_{\nu}(k a)+B_{\ell} Y_{\nu}(k a)=0 .
$$

In the second condition, one can develop in Fourier series the scattering amplitude $f(\varphi, k)=\Sigma_{\ell \in Z} e^{i \ell \varphi} f_{\ell}$, and the plane wave $e^{i k x}$ (which can be written in terms of Bessel functions):

$$
\begin{aligned}
& e^{i k r \cos (\varphi)}+f(\varphi, k) \frac{e^{i k r}}{\sqrt{r}} \\
& =\sum_{\ell \in Z} e^{i \ell \varphi}\left[i^{|\ell|} J_{|\ell|}(k r)+f_{\ell} \frac{e^{i k r}}{\sqrt{r}}\right] \\
& \sim \sum_{\ell \in Z} e^{i \ell \varphi}\left[\frac{e^{i k r}}{\sqrt{r}}\left(\frac{i^{|\ell|}}{\sqrt{2 \pi k}} e^{-i(\pi|\ell| / 2+\pi / 4)}+f_{\ell}\right)\right. \\
& \left.+\frac{e^{-i k r}}{\sqrt{r}}\left(\frac{i^{|\ell|}}{\sqrt{2 \pi k}} e^{i(\pi|\ell| / 2+\pi / 4)}\right)\right],
\end{aligned}
$$

where we have replaced the asymptotic expression of Bessel functions inside the series.

Comparing the terms in Eq. (60) with the asymptotic expression of $\chi_{l}^{(0)}(r)$ in Eq. (57) for large values of $k r$ [see Eq. (41)] we get the following equations:

$$
\begin{aligned}
i^{|\ell|} e^{-i(\pi|\ell| / 2+\pi / 4)}+\sqrt{2 \pi k} f_{\ell} & =\left(A_{\ell}-i B_{\ell}\right) e^{-i(\pi \nu / 2+\pi / 4)}, \\
i^{|\ell|} e^{i(\pi|\ell| / 2+\pi / 4)} & =\left(A_{\ell}+i B_{\ell}\right) e^{i(\pi \nu / 2+\pi / 4)} .
\end{aligned}
$$

The solution to the set of equations (59), (61) and (62) is

$$
\begin{aligned}
& A_{\ell}=i e^{-(i / 2) \pi(\nu-2 \ell)} \frac{Y_{\nu}(k a)}{H_{\nu}^{(1)}(k a)}, \\
& B_{\ell}=-i e^{-(i / 2) \pi(\nu-2 \ell)} \frac{J_{\nu}(k a)}{H_{\nu}^{(1)}(k a)}, \\
& f_{\ell}=-\frac{e^{-i(\pi / 4)}}{\sqrt{2 \pi k}}\left[1+e^{-i \pi(\nu-\ell)} \frac{H_{\nu}^{(2)}(k a)}{H_{\nu}^{(1)}(k a)}\right],
\end{aligned}
$$

where the $H_{\nu}^{(1,2)}(z)$ are the Hankel functions.

From Eq. (65) one can easily extract the phase shifts. Indeed, from scattering theory [19], one knows that the scattering amplitude for the $\ell$ th partial wave is

$$
f_{\ell}=\frac{e^{-i(\pi / 4)}}{\sqrt{2 \pi k}}\left(e^{2 i \delta_{\ell}}-1\right)
$$

Then, in the present case

$$
e^{2 i \delta_{\ell}}=(-1)^{\ell+1} e^{-i \pi \nu} \frac{H_{\nu}^{(2)}(k a)}{H_{\nu}^{(1)}(k a)},
$$

which provides an exact expression for the $S$-matrix [13].

One can check the consistency of our approach by evaluating the limit $a \rightarrow 0$. In this case $f_{\ell}$ reduces to

$$
f_{0, \ell}=\frac{e^{-i \frac{\pi}{4}}}{\sqrt{2 \pi k}}\left[e^{-i \pi(\nu-\ell)}-1\right],
$$

or, equivalently,

$$
\delta_{\ell}=\frac{\pi}{2}(|\ell|-\nu)
$$

Equations (67)-(69) are in agreement with other derivations found in the literature $[13,20]$. Notice that the phase shifts $\delta_{\ell}$ do not tend to 0 for $\ell \rightarrow \pm \infty$; instead, they approach nonvanishing constants (see the discussion in [17]).

In order to compute the differential cross section one must get, first, the total scattering amplitude, i.e., we must evaluate the sum

$$
f(\varphi, k)=\sum_{\ell=-\infty}^{\infty} f_{\ell} e^{i \ell \varphi} .
$$

The explicit calculation of Eq. (70) involves several technical and conceptual difficulties which have been a source of controversy in the past [21]. 
First, let us consider the case $a=0$. Making use of Eq. (68), the total amplitude becomes

$$
f_{0}(\varphi, k)=\frac{e^{-i(\pi / 4)}}{\sqrt{2 \pi k}} \sum_{\ell=-\infty}^{\infty} e^{i \ell \varphi}\left(-1+e^{-i \pi \nu}(-1)^{\ell}\right) .
$$

The first term in Eq. (71) is

$$
\begin{aligned}
\frac{e^{-i(\pi / 4)}}{\sqrt{2 \pi k}} \sum_{\ell=-\infty}^{\infty} e^{i \ell \varphi}(-1) & =-\frac{e^{-i(\pi / 4)}}{\sqrt{2 \pi k}} 2 \pi \delta[\varphi] \\
& =-\sqrt{\frac{2 \pi}{i k}} \delta[\varphi] .
\end{aligned}
$$

For the second one we get (see Appendix B for details)

$$
\begin{aligned}
& \frac{e^{-i(\pi / 4)}}{\sqrt{2 \pi k}} \sum_{\ell=-\infty}^{\infty} e^{i \ell \varphi}\left(e^{-i \pi \nu}(-1)^{\ell}\right) \\
& \quad=\frac{e^{-i(\pi / 4)}}{\sqrt{2 \pi k}}\left\{2 \pi \cos \left(\frac{\Phi}{2}\right) \delta[\varphi]+2 i \sin \left(\frac{\Phi}{2}\right) \mathcal{P}\left[\frac{e^{i\left(\ell_{0}+1\right) \varphi}}{1-e^{i \varphi}}\right]\right\},
\end{aligned}
$$

where $l_{0}$ is the integer part of $\Phi / 2 \pi$ and $\mathcal{P}[F(\varphi)]$ denotes the principal value of $F(\varphi)$.

Finally, the scattering amplitude becomes

$$
\begin{aligned}
f_{0}(\varphi, k)= & \sqrt{\frac{2 \pi}{i k}}\left\{\left[\cos \left(\frac{\Phi}{2}\right)-1\right] \delta[\varphi]\right. \\
& \left.+\frac{i}{\pi} \sin \left(\frac{\Phi}{2}\right) \mathcal{P}\left[\frac{e^{i\left(l_{0}+1\right) \varphi}}{1-e^{i \varphi}}\right]\right\} \\
= & \sqrt{\frac{2 \pi}{i k}}\left\{\left[\cos \left(\frac{\Phi}{2}\right)-1\right] \delta[\varphi]\right. \\
& \left.+\frac{i}{\pi} \sin \left(\frac{\Phi}{2}\right)\left(\mathcal{P}\left[\frac{i}{\varphi}\right]+\left[\frac{e^{i\left(l_{0}+1\right) \varphi}}{1-e^{i \varphi}}-\frac{i}{\varphi}\right]\right)\right\}
\end{aligned}
$$

Notice that $f_{0}(\varphi, k)$ vanishes for $\Phi=4 \pi n$, with $n$ integer. For these flux values the particles are not scattered at all by the zero radius solenoid in the commutative case.

This formula coincides with Eq. (4.11) of [13], where it was obtained following a different procedure. The interpre- tation of the forward scattering term in Eq. (74), in the context of the construction of the scattering matrix, is considered in that reference. Many authors have discussed the presence or not of this forward scattering singular term in the total scattering amplitude (see, for example [17]). It is not present in the original derivation by Aharonov and Bohm [5], and can be also avoided making use of an analytic regularization as in [18]. However, as previously pointed out, in the present work we are interested in the calculation of the differential cross section for scattering angles different from zero, where different approaches coincide. This justifies the partial waves analysis we performed.

The calculation of the differential cross section is now immediate. Indeed, for $\varphi \neq 0$ we have

$$
\frac{d \sigma}{d \varphi}=\left|f_{0}(\varphi, k)\right|^{2}=\frac{\sin ^{2}\left[\frac{\Phi}{2}\right]}{2 \pi k \sin ^{2}\left[\frac{\varphi}{2}\right]}
$$

which is the usual Aharonov-Bohm differential cross section [5], vanishing for $\Phi=2 \pi n$, with $n$ integer.

If the radius of the solenoid is different from zero ( $a$ $>0$ ), one can similarly isolate the singular contributions to the total scattering amplitude $f(\varphi, k)$, coming from large values of $\ell$ (or equivalently, from large values of $\nu$ ). Using appropriate large order expansions for the Hankel functions, one finds that the coefficient $f_{\ell}$ is given in this case by the RHS of Eq. (68) plus terms rapidly decreasing with $\ell$, which lead to absolutely convergent series (summing up to continuous functions of $\varphi$ ). Therefore, the singular terms found in Eq. (74) for $f_{0}(\varphi, k)$ (those containing $\delta[\varphi]$ and $\mathcal{P}[i / \varphi]$ ) are also present in $f(\varphi, k)$.

\section{FIRST ORDER NONCOMMUTATIVE CORRECTIONS TO THE SCATTERING AMPLITUDE}

In this section we calculate the first order $\left(\theta^{1}\right)$ perturbative correction to the scattering amplitude $f(\varphi, k)$. This will allow us to find the first noncommutative (singular) corrections to the differential cross section.

In doing so, we must evaluate $\Psi_{1}(\mathbf{x})$ in Eq. (49), with $\Psi_{0}(\mathbf{x})$ given in Eqs. (57), (63) and (64), and $G\left(\mathbf{x}, \mathbf{x}^{\prime}\right)$ given in Eqs. (51), (55) and (56).

Taking into account that $\hat{H}_{0}$ and $\hat{H}_{1}$ are diagonal in $\ell$, we can write $\Psi_{1}(r, \varphi)=\Sigma_{\ell} e^{i \ell \varphi} \chi_{\ell}^{(1)}(r)$ to get

$$
\begin{aligned}
\chi_{\ell}^{(1)}(r) & =-\int_{a}^{\infty} g_{\ell}(r, s)\left(\hat{h}_{\ell, 1} \chi_{\ell}^{(0)}(s)\right) s d s \\
& =-C_{0}\left[\chi_{\ell}^{(\infty)}(r) \int_{a}^{r} \chi_{\ell}^{(a)}(s)\left(\hat{h}_{\ell, 1} \chi_{\ell}^{(0)}(s)\right) s d s+\chi_{\ell}^{(a)}(r) \int_{r}^{\infty} \chi_{\ell}^{(\infty)}(s)\left(\hat{h}_{\ell, 1} \chi_{\ell}^{(0)}(s)\right) s d s\right],
\end{aligned}
$$


with $\chi_{\ell}^{(\infty)}(r)$ and $\chi_{\ell}^{(a)}(r)$ given in Eq. (54), and $\hat{h}_{\ell, 1}$ given in Eq. (33).

Since we are interested in the noncommutative corrections to the scattering amplitude, we should consider the asymptotic behavior of $\chi_{\ell}^{(1)}(r)$ for $r \rightarrow \infty$. The expansions for large arguments of Bessel function in Eq. (41) allow us to see that the second term in the brackets in the RHS of Eq. (76) decreases faster than the first one, and can be discarded.

For arbitrary $a>0$, the integrand in the first term is too complicated to give a closed solution to this integral, and some simplification is necessary. For this reason, we will analyze it only in the $a \rightarrow 0$ limit.

In this limit, straightforward calculations lead to

$$
C_{0} \chi_{\ell}^{(a)}(r) \rightarrow{ }_{a \rightarrow 0}-\frac{i \pi}{2} J_{\nu}(k r)
$$

and

$$
\begin{aligned}
\hat{h}_{\ell, 1} \chi_{\ell}^{(0)}(s) \rightarrow & { }_{a \rightarrow 0} \frac{(-1)^{\ell} e^{-(1 / 2) i \pi \nu}}{8 \pi^{3} s^{4}}\left\{\pi^{2}(4 \pi \ell\right. \\
& -\Phi) k s\left(J_{\nu+1}(k s)-J_{\nu-1}(k s)\right)+\left(8 \pi^{3} \ell^{3}\right. \\
& \left.\left.-8 \pi^{2} \ell^{2} \Phi+4 \pi \ell \Phi^{2}-\Phi^{3}\right) J_{\nu}(k s)\right\},
\end{aligned}
$$

while $\chi_{\ell}^{(\infty)}(r)$ does not depend on $a$.

Then, for $\nu>1$ [22] the coefficient of $\chi_{\ell}^{(\infty)}(r)$ in the RHS of Eq. (76) reduces in this approximation to

$$
\begin{aligned}
& \frac{i}{16 \pi^{2}}(-1)^{\ell} e^{-(i / 2) \pi \nu} k^{2} \\
& \times\left[\frac{\left(8 \pi^{3} \ell^{3}-8 \pi^{2} \ell^{2} \Phi-8 \pi^{3} \ell+4 \pi \ell \Phi^{2}+2 \pi^{2} \Phi-\Phi^{3}\right)}{4 \nu\left(\nu^{2}-1\right)}\right],
\end{aligned}
$$

an expression in which we must distinguish two cases, namely

$$
\ell>\frac{\Phi}{2 \pi} \quad \text { and } \quad \ell \leqslant \frac{\Phi}{2 \pi} .
$$

If $\ell \geqslant l_{0}+1>\Phi / 2 \pi$ (where $l_{0}$ is the integer part of $\Phi / 2 \pi$ ), then $\nu=\ell-\Phi / 2 \pi$, and Eq. (79) becomes

$$
i(-1)^{\ell} e^{-(i / 4)(2 \pi \ell-\Phi)} \pi k^{2}\left(\frac{1}{8}+\frac{\Phi}{16 \pi \ell}+\mathcal{O}\left(\ell^{-2}\right)\right) .
$$

Now, taking into account that, for $r \rightarrow \infty$,

$$
\chi_{\ell}^{(\infty)}(r)=H_{\nu}^{(1)}(k r) \sim \sqrt{\frac{2}{\pi k}} e^{-i(\pi / 2)(\ell-\Phi / 2 \pi)-i(\pi / 4)} \frac{e^{i k r}}{\sqrt{r}},
$$

we get the first perturbative correction to $f_{\ell}$ in Eq. (70) as

$$
\theta f_{1, \ell}=\frac{\theta}{4} \sqrt{\frac{i \pi}{2}} e^{i(\Phi / 2)} k^{3 / 2}\left(1+\frac{\Phi}{2 \pi \ell}+\mathcal{O}\left(\ell^{-2}\right)\right) .
$$

Multiplying this expression by $e^{i \ell \varphi}$ and summing on $\ell$ from $\ell_{0}+1$ to $\infty$, we obtain the following contribution to the scattering amplitude:

$$
\begin{aligned}
& \frac{\theta}{4} \sqrt{\frac{i \pi}{2}} e^{i(\Phi / 2)} k^{3 / 2}\left(\pi \delta[\varphi]+\mathcal{P}\left[\frac{1}{1-e^{i \varphi}}\right]-\frac{\Phi}{2 \pi} \log [1\right. \\
& \left.\left.-e^{i(i \epsilon+\varphi)}\right]+\cdots\right)
\end{aligned}
$$

where the $\epsilon \rightarrow 0^{+}$limit is understood, and the dots stand for continuous functions of $\varphi$.

For the case $\ell \leqslant \ell_{0} \leqslant \Phi / 2 \pi$, we have $\nu=\Phi / 2 \pi-\ell$, and a similar calculation (where the sum on $\ell$ is taken from $-\infty$ to $\ell_{0}$ ) leads to the following contribution to the scattering amplitude:

$$
\begin{gathered}
\frac{\theta}{4} \sqrt{\frac{i \pi}{2}} e^{-i(\Phi / 2)} k^{3 / 2}\left(\pi \delta[\varphi]-\mathcal{P}\left[\frac{1}{1-e^{-i \varphi}}\right]\right. \\
\left.-\frac{\Phi}{2 \pi} \log \left[1-e^{-i(-i \epsilon+\varphi)}\right]+\cdots\right)
\end{gathered}
$$

where, again, the limit $\epsilon \rightarrow 0^{+}$is understood and the dots represent continuous functions of $\varphi$.

Therefore, at first order in $\theta$ the scattering amplitude is corrected by the addition of

$$
\begin{aligned}
\theta f^{(1)}(\varphi, k)= & \frac{\theta}{4} \sqrt{\frac{i \pi}{2}} k^{3 / 2}\left\{2 \pi \cos \left(\frac{\Phi}{2}\right) \delta[\varphi]\right. \\
& +i \cos \left(\frac{\Phi-\varphi}{2}\right) \mathcal{P}\left[\frac{1}{\sin \left(\frac{\varphi}{2}\right)}\right] \\
& -\frac{\Phi}{2 \pi} e^{-i(\Phi / 2)} \log \left[1-e^{-i(-i \epsilon+\varphi)}\right] \\
& -\frac{\Phi}{2 \pi} e^{i(\Phi / 2)} \log \left[1-e^{i(i \epsilon+\varphi)}\right]+\cdots
\end{aligned} .
$$

In conclusion, as the incident particles are very energetic and the scattering angle is very small, the main contributions to the total scattering amplitude $f(\varphi, k)$ are given by [23] 


$$
\begin{aligned}
f(\varphi, k)= & \left\{\sqrt{\frac{2 \pi}{i k}}\left[\cos \left(\frac{\Phi}{2}\right)-1\right]\right. \\
& \left.+\theta \frac{\pi}{2} \sqrt{\frac{i \pi}{2}} k^{3 / 2} \cos \left(\frac{\Phi}{2}\right)\right\} \delta[\varphi] \\
& +\left\{-\sqrt{\frac{2}{i \pi k}} \sin \left(\frac{\Phi}{2}\right)+\theta \frac{i}{2} \sqrt{\frac{i \pi}{2}} k^{3 / 2} \cos \left(\frac{\Phi}{2}\right)\right\} \mathcal{P}\left[\frac{1}{\varphi}\right]
\end{aligned}
$$

$$
\begin{aligned}
& -\frac{\theta}{4} \sqrt{\frac{i}{2 \pi}} k^{3 / 2} \Phi \cos \left(\frac{\Phi}{2}\right) \log (\varphi) \\
& + \text { continuous functions of } \varphi
\end{aligned}
$$

Notice that the most singular terms in the scattering amplitude, which are $\sim k^{-1 / 2}$, are corrected by noncommutative terms $\sim \theta k^{3 / 2}$. Moreover, for $\Phi=4 \pi n$, with $n$ integer, the 0th order singular terms in the amplitude vanish, contrary to the noncomutative corrections, which are different from zero.

For small angles $\varphi \neq 0$, the dominant term in the amplitude is $\sim 1 / \varphi$. Then, for the differential cross section we have

$$
\begin{aligned}
\frac{d \sigma}{d \varphi}= & \left\{\frac{2}{\pi k} \sin ^{2}\left(\frac{\Phi}{2}\right)+\theta \frac{k}{2} \sin (\Phi)+\theta^{2} \frac{\pi}{8} k^{3} \cos ^{2}\left(\frac{\Phi}{2}\right)\right\} \frac{1}{\varphi^{2}} \\
& + \text { less singular terms. }
\end{aligned}
$$

Now, if the magnetic flux is quantized as $\Phi=2 \pi n$, with $n$ integer, the differential cross section at small angles is dominated by noncommutative effects,

$$
\frac{d \sigma}{d \varphi}=\theta^{2} \frac{\pi k^{3}}{8 \varphi^{2}}+\text { less singular terms }
$$

It is interesting to note that, contrary to the usual AharonovBohm effect, in the noncommutative case the differential scattering cross section is different from zero when the magnetic flux is quantized.

Apparently, this correction $\left(\sim \theta^{2}\right)$ could be relevant at high energies. This simple formula will allow us to extract interesting physical information, as we will see in the next section.

\section{PHENOMENOLOGICAL ESTIMATIONS FOR SPATIAL NONCOMMUTATIVE EFFECTS}

As mentioned in the Introduction, the Aharonov-Bohm effect is an important mechanism to explain other physical phenomena. This point of view has been used in the past, and some applications of this idea are cosmic strings and GUT [8], anyons [24] and also three-dimensional gravity [20].

In this section we will analyze experimental possibilities of detecting noncommutative signals via the AharonovBohm effect. Our numerical estimations - as we will see below-show that these relics could be explored in particle physics experiments involving energies between 200 and 300 $\mathrm{GeV}$, if the present bound for $\theta$ is correct.

In order to estimate a bound for the $\theta$ parameter, first we note that, since noncommutative effects are tiny, the corrections to the differential cross section could be, typically, of the order of the cross section for neutrino events $\sim 10^{-3} \mathrm{nb}$. If we choose the scattering angle between 1 and 2 degrees, and take an energy $\sim 200 \mathrm{GeV}$ as the highest possible presently available for electrons, then we find

$$
\theta=\left[\left(\frac{8 \varphi^{2}}{\pi k^{3}}\right) \frac{d \sigma}{d \varphi}\right]^{1 / 2} \sim[10 \mathrm{TeV}]^{-2}
$$

which is in agreement with the bound given in [9].

Thus, precise measurements of the differential cross section for small angles could give us information about spatial noncommutativity.

\section{CONCLUSIONS}

Three relevant properties of the remarkable phenomenon of noncommutative Aharonov-Bohm effect have been found in the present paper:

Pattern fringes can appear even when the magnetic flux is quantized, contrary to the commutative case.

The differential cross section, given by Eq. (90), is different from zero when the magnetic flux is quantized.

Our results allow for an estimation of a bound for the noncommutative parameter $\theta$, which is in agreement with [9].

The first property, in principle, could be verified in a Tonomura like experiment, if an appropriate incident electron beam is available. Our estimations suggest, however, that the incident electron beam energy should be much larger than the energy reached in these experiments [25]. Thus, an experimental verification should be searched in high energy physics experiments and, specially, by measuring differential cross sections for small angles.

\section{ACKNOWLEDGMENTS}

This work has been partially supported by the grants 1010596, 1010976, 7010976 and 3000005 from FondecytChile, and also by the grantC-13398/6 from Fundacion Andes. H.F. also acknowledges support from CONICET (grant 0459/98) and UNLP (grant 11/X298), Argentina.

\section{APPENDIX A: NOTE ON THE RELATIVISTIC AHARONOV-BOHM EFFECT}

In this appendix, we would like to discuss some implications of the relativistic Aharonov-Bohm effect.

From Ref. [6] one can see that the Green's function associated to the usual Aharonov-Bohm effect is given by 


$$
G\left[x, x^{\prime}\right]=\sum_{n=-\infty}^{\infty}(-i)^{|n+\varphi|} \exp [-i(n+\Phi)] F_{|n+\Phi|}
$$

where $\Phi$ is the magnetic flux and the function $F_{|n+\Phi|}$ for the nonrelativistic case is

$$
\begin{aligned}
F_{|n+\Phi|}= & \frac{m}{2 \pi i} \exp \left[\frac{2 m i}{\tau}\left(r^{2}+r^{\prime 2}\right)\right] \\
& \times J_{|n+\Phi|}\left(\frac{m r r^{\prime}}{\tau}\right),
\end{aligned}
$$

where $\tau=t-t^{\prime}$ and $J_{\alpha}$ are Bessel functions. For the relativistic case the calculation is similar. Indeed, after using the proper-time gauge the function $F_{|n+\Phi|}$ becomes

$$
\begin{aligned}
F_{|n+\Phi|}= & \int d^{2} p \int_{0}^{\infty} d T \exp \left[i p_{\mu} \Delta x^{\mu}-\frac{T}{2}\left(p^{2}+m^{2}\right)\right] \\
& \times J_{|n+\Phi|}\left(\frac{r r^{\prime}}{T}\right),
\end{aligned}
$$

where $T=N(0)\left(t-t^{\prime}\right)$ with $N(0)$ the einbein.

If we use the Poisson summation formula, then in both the relativistic as well as in the nonrelativistic case, the Green's function is

$$
G\left[x, x^{\prime}\right]=\sum_{n=-\infty}^{\infty} e^{2 i \pi n \Phi} K_{n},
$$

where $K_{n}$ is defined as

$$
K_{n}=\int_{-\infty}^{\infty} d \omega(-i)^{|\omega|} e^{-i \omega \Phi} F_{|\omega|},
$$

and, as a consequence, the wave function becomes

$$
\psi(x)=\sum_{n=-\infty}^{\infty} e^{2 i \pi n \Phi} \varphi_{n}(x)
$$

with

$$
\varphi_{n}(x)=\int d y G_{n}[x, y] \psi(y)
$$

being $\varphi_{n}$ and $G_{n}[x, y]$, respectively, the wave and Green's functions for the $n$th homotopy class [26].

Thus, from Eq. (A6) one sees that the relativistic character of the system is contained in $K_{n}$ and only the exponential factor, which does not depend on the energy, is responsible for the fringe pattern. This result reflects the topological nature of the commutative Aharonov-Bohm effect. However, our formula (14) shows us that the noncommutative Ahararonov-Bohm effect is radically different because the fringe pattern must change when the electrons are getting higher energies.
APPENDIX B: DERIVATION OF EQ. (73)

In this appendix we show that

$$
\begin{aligned}
\sum_{\ell=-\infty}^{\infty} e^{i \ell \varphi} e^{-i \pi \nu}(-1)^{\ell}= & 2 \pi \cos \left(\frac{\Phi}{2}\right) \delta[\varphi] \\
& +2 i \sin \left(\frac{\Phi}{2}\right) \mathcal{P}\left[\frac{e^{i\left(\ell_{0}+1\right) \varphi}}{1-e^{i \varphi}}\right]
\end{aligned}
$$

where $\ell_{0}$ is the integer part of $\Phi / 2 \pi$. First, notice that $e^{-i \pi \nu}(-1)^{\ell}=e^{i \pi(|\ell|-|\ell-\Phi / 2 \pi|)}$, since the exponents coincide modulo $2 \pi$. Moreover, if $\ell \geqslant \ell_{0}+1$ then $|\ell-\Phi / 2 \pi|=\ell$ $-\Phi / 2 \pi$, while if $\ell \leqslant \ell_{0}$ then $|\ell-\Phi / 2 \pi|=-\ell+\Phi / 2 \pi$.

Therefore, we can split the series in Eq. (B1) to write

$$
\begin{aligned}
& \sum_{\ell=-\infty}^{\infty} e^{i \ell \varphi} e^{i \pi(|\ell|-|\ell-\Phi / 2 \pi|)} \\
& =\sum_{\ell=-\infty}^{\ell_{0}} e^{i \ell \varphi} e^{-i \pi(\Phi / 2 \pi)}+\sum_{\ell=\ell_{0}+1}^{\infty} e^{i \ell \varphi} e^{i \pi(\Phi / 2 \pi)} \\
& \equiv e^{-i(\Phi / 2)} e^{i \ell} \lim _{\epsilon \rightarrow 0} \sum_{\ell=0}^{\infty} e^{-i \ell(\varphi-i \epsilon)}+e^{i(\Phi / 2)} e^{i\left(\ell_{0}+1\right) \varphi} \\
& \quad \times \lim _{\epsilon \rightarrow 0} \sum^{\infty} e^{i \ell(\varphi+i \epsilon)},
\end{aligned}
$$

where we have introduced the positive parameter $\epsilon$ to properly define these sums.

Now, the evaluation goes in the standard way. For the first series we have

$$
e^{-i(\Phi / 2)} e^{i \ell_{0} \varphi} \lim _{\epsilon \rightarrow 0} \sum_{\ell=0}^{\infty} e^{-i \ell(\varphi-i \epsilon)}
$$

$$
\begin{aligned}
= & \lim _{\epsilon \rightarrow 0} \frac{e^{-i(\Phi / 2)} e^{i \ell_{0} \varphi}}{1-e^{-i(\varphi-i \epsilon)}} \\
= & -i e^{-i(\Phi / 2)} e^{i\left(\ell_{0}+1\right) \varphi}\left(i \pi \delta\left[i\left(1-e^{i \varphi}\right)\right]\right. \\
& \left.+\mathcal{P}\left[\frac{1}{i\left(1-e^{i \varphi}\right)}\right]\right) \\
= & \pi e^{-i(\Phi / 2)} \delta[\varphi]-e^{-i(\Phi / 2)} \mathcal{P}\left[\frac{e^{i\left(\ell_{0}+1\right) \varphi}}{1-e^{i \varphi}}\right],
\end{aligned}
$$

where $\mathcal{P}[\cdots]$ means principal value. 
The second series in the RHS of Eq. (B2) is evaluated in a similar way,

$$
\begin{aligned}
& e^{i(\Phi / 2)} e^{i\left(\ell_{0}+1\right) \varphi} \lim _{\epsilon \rightarrow 0} \sum_{\ell=0}^{\infty} e^{i \ell(\varphi+i \epsilon)} \\
& =\lim _{\epsilon \rightarrow 0} \frac{e^{i(\Phi / 2)} e^{i\left(\ell_{0}+1\right) \varphi}}{1-e^{i(\varphi+i \epsilon)}} \\
& =i e^{i(\Phi / 2)} e^{i\left(\ell_{0}+1\right) \varphi}\left(-i \pi \delta\left[i\left(1-e^{i \varphi}\right)\right]\right. \\
& \left.+\mathcal{P}\left[\frac{1}{i\left(1-e^{i \varphi}\right)}\right]\right)
\end{aligned}
$$$$
=\pi e^{i(\Phi / 2)} \delta[\varphi]+e^{i(\Phi / 2)} \mathcal{P}\left[\frac{e^{i\left(\ell_{0}+1\right) \varphi}}{1-e^{i \varphi}}\right]
$$

Collecting both results one finally obtains

$$
\begin{aligned}
& \sum_{\ell=-\infty}^{\infty} e^{i \ell \varphi} e^{-i \pi \nu}(-1)^{\ell} \\
& \quad=2 \pi \cos \left(\frac{\Phi}{2}\right) \delta[\varphi]+2 i \sin \left(\frac{\Phi}{2}\right) \mathcal{P}\left[\frac{e^{i\left(\ell_{0}+1\right) \varphi}}{1-e^{i \varphi}}\right] .
\end{aligned}
$$

[1] A. Connes, M. Douglas, and A.S. Schwarz, J. High Energy Phys. 02, 003 (1998); N. Seiberg and E. Witten, ibid. 09, 032 (1999).

[2] The literature is very extensive; some references are T. Filk, Phys. Lett. B 376, 53 (1996); R. Gopakumar, J. Maldacena, S. Minwalla, and A. Strominger, J. High Energy Phys. 06, 036 (2000); L. Alvarez-Gaumé and S. Wadia, Phys. Lett. B 501, 319 (2001); M. Hayakawa, ibid. 476, 431 (2000); C.P. Martin and F. Ruiz, Nucl. Phys. B597, 197 (2001); A. Armoni, ibid. B593, 229 (2001); J. Gomis and T. Mehen, ibid. B591, 265 (2000); I. Mociou, M. Popelov, and R. Roiban, Phys. Lett. B 489, 390 (2000); C.E. Carlson, C.D. Carone, and R.F. Lebed, ibid. 518, 201 (2001); K. Valavano, Class. Quantum Grav. 17, 4491 (2001); C. Duval and P.A. Horváthy, J. Phys. A 34, 10097 (2001); Phys. Lett. B 479, 284 (2000); Z. Guralnik, R. Jackiw, S.Y. Pi, and A.P. Polychronakos, ibid. 517, 450 (2001); M. Lubo, Phys. Rev. D 65, 066003 (2002); I. Mocioiu, M. Popelov, and R. Roiban, Phys. Lett. B 489, 390 (2000); J. Gamboa, M. Loewe, F. Méndez, and J.C. Rojas, hep-th/0106125; J. Gamboa, M. Loewe, and J.C. Rojas, Phys. Rev. D 64, 067901 (2001); J. Gamboa, M. Loewe, F. Méndez, and J.C. Rojas, Mod. Phys. Lett. A 16, 2075 (2001); A.P. Polychronakos, J. High Energy Phys. 11, 008 (2000); S. Gubser and M. Rangamani, ibid. 05, 041 (2001); V.P. Nair and A.P. Polychronakos, Phys. Lett. B 505, 267 (2001); D. Karabali, V.P. Nair, and A.P. Polychronakos, Nucl. Phys. B627, 565 (2002); O.F. Dayi and L.T. Kelleyane, hep-th/0202062; O.F. Dayi and A. Jellal, hep-th/0111267; P.A. Horvathy, hep-th/0201007; D.H. Correa, G.S. Lozano, E.F. Moreno, and F.A. Schaposnik, Phys. Lett. B 515, 206 (2001); H.R. Christiansen and F.A. Schaposnik, Phys. Rev. D 65, 086005 (2002); R. Iengo and R. Ramachandran, J. High Energy Phys. 02, 017 (2002).

[3] C.A. Mead and D.G. Truhlar, J. Chem. Phys. 70, 2284 (1979); M. Berry, Proc. R. Soc. London Ser. A 392, 45 (1984).

[4] See, e.g., A. Shapere and F. Wilczek, Geometric Phases in Physics (World Scientific, Singapore, 1989).

[5] Y. Aharonov and D. Bohm, Phys. Rev. 115, 485 (1958).
[6] J. Gamboa and V.O. Rivelles, J. Phys. A 24, L659 (1991).

[7] M. Peshkin, I. Talmi, and L.J. Tassie, Ann. Phys. (N.Y.) 16, 426 (1961).

[8] M.G. Alford and F. Wilczek, Phys. Rev. Lett. 62, 1017 (1988).

[9] S. Carroll, J. Harvey, V.A. Kostelecky, C.D. Lane, and T. Okamoto, Phys. Rev. Lett. 87, 141601 (2001).

[10] M. Cheichian, A. Demishev, P. Presnajder, M. Cheikh-Jabbari, and A. Tureanu, Phys. Lett. B 527, 149 (2002).

[11] J. Gamboa, M. Loewe, and J.C. Rojas, hep-th/0101081.

[12] A different expression was obtained in [10], although later it was corrected by these authors who agree with our previous result [11].

[13] S.N.M. Ruijsenaars, Ann. Phys. (N.Y.) 146, 1 (1983).

[14] H. Falomir and P.A.G. Pisani, J. Phys. A 34, 4143 (2001).

[15] P. de S. Gerbert and R. Jackiw, Commun. Math. Phys. 124, 229 (1989).

[16] A. Yelnikov, hep-th/0112134.

[17] C.R. Hagen, Phys. Rev. D 41, 2015 (1990).

[18] P. Giacconi, F. Maltoni, and R. Soldati, Phys. Rev. D 53, 952 (1996).

[19] See, e.g., J. Taylor, Scattering Theory (Wiley, New York, 1972).

[20] See, e.g., S. Deser and R. Jackiw, Commun. Math. Phys. 118, 495 (1988).

[21] E.L. Feinberg, Sov. Phys. Usp. 5, 753 (1963); E. Corinaldesi and F. Rafeli, Am. J. Phys. 46, 1255 (1978).

[22] For $\nu \leqslant 1$, the first integral in the RHS of Eq. (76) is divergent in the $a \rightarrow 0$ limit, and one should keep $a>0$ in these two (or at most three) terms. For the consideration of the most general acceptable boundary conditions at $r=0$, see for example the study of the self adjoint extensions of the Hamiltonian discussed in [18]. Since we are actually interested in the singular contributions to the scattering amplitude for small angles, coming from the large $|\ell|$ behavior of $\chi_{\ell}^{(1)}(r)$, we will not worry about these terms (whose contributions to $\Psi_{1}$ are smooth, continuous function of $\varphi$ ). 
[23] The reader could note here that this argument is reminiscent of the eikonal approximation, see, e.g., J. J. Sakurai, Modern Quantum Mechanics (Addison-Wesley, Redwood City, CA, 1994).

[24] See, e.g., F. Wilczek, Fractional Statistics and Anyon Superconductivity (World Scientific, Singapore, 1989).
[25] A. Tonomura, Phys. Rev. Lett. 48, 1443 (1983); see also M. Peshkin and A. Tonomura, The Quantum Hall Effect (SpringerVerlag, Berlin, 1989).

[26] M.G. Laidlaw and C.M. de Witt, Phys. Rev. D 3, 1375 (1971); L.S. Schulman, Phys. Rev. 176, 1558 (1968). 